\title{
The role of brain magnetic resonance imaging on the timing of antiepileptic drugs withdrawal following mesial temporal lobe epilepsy surgery
}

Miad Albalawi, MD, Fawzi Babtain, MD, Saleh Baeesa, MD, FRCSC, Youssef Al-Said, MD, FRCPC, Khalid Alqadi, MD.

\begin{abstract}

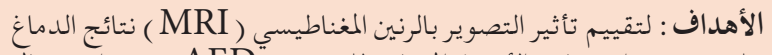

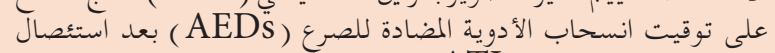

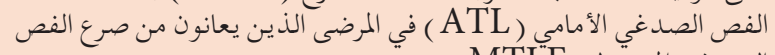
الصدغي المتوسط (MTLE)

المنهجية : لقد أجرينا مراجعة بأثر رجعي في مستشفى الملك فيصل

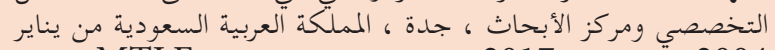

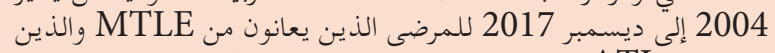

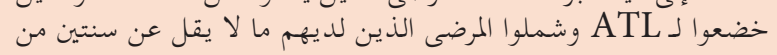

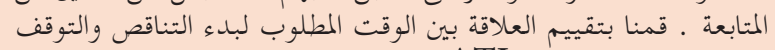

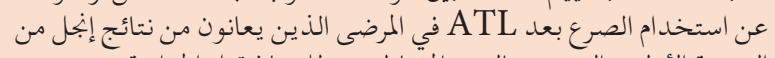

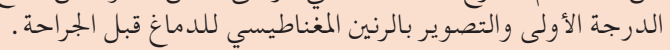

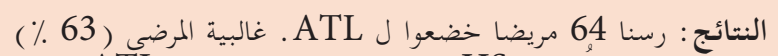

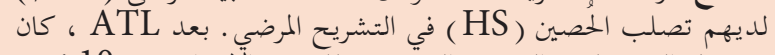

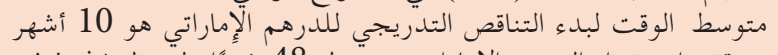

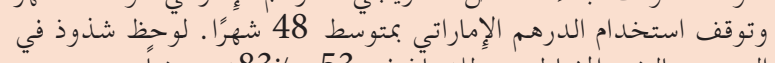

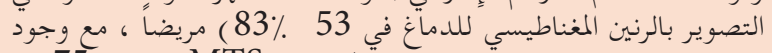

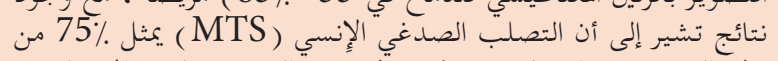

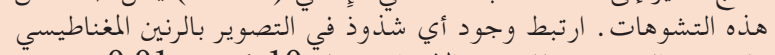

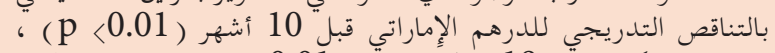

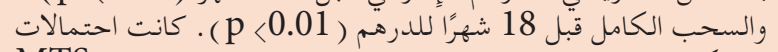

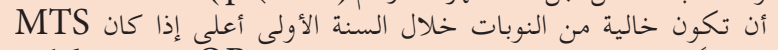

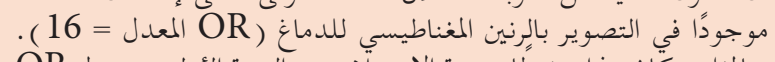

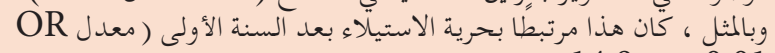

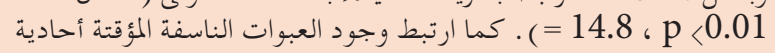

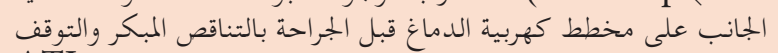

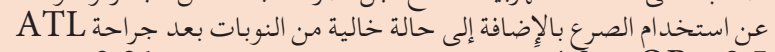

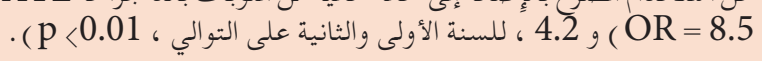

الحلاصة : كان المرضى الذين يعانون من نتائج غير طبيعية للتصوير بالرنين المباطين

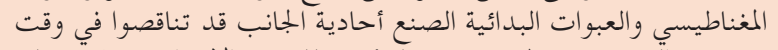

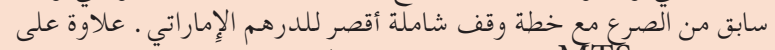

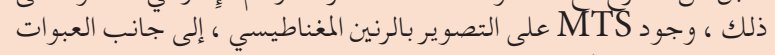

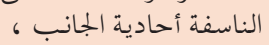

Objectives: To assess the influence of magnetic resonance imaging (MRI) brain findings on the timing of antiepileptic drugs (AEDs) withdrawal following anterior temporal lobectomy (ATL) in patients with mesial temporal lobe epilepsy (MTLE).
\end{abstract}

Methods: We conducted a retrospective chart review at King Faisal Specialist Hospital and Research Centre, Jeddah, Saudi Arabia from Jan, 2004 - Dec, 2017 of patients with MTLE who underwent ATL and included patients who had a minimum of 2 years of follow-up. We evaluated the association between the time required to start tapering and discontinuing AEDs after ATL in patients with Engel class I outcomes and their preoperative brain MRI.

Results: We studied 64 patients who underwent ATL. The majority of patients (63\%) had hippocampal sclerosis (HS) on histopathology. Following ATL, the mean time to start tapering AEDs was 10 months and AEDs were discontinued at a mean of 48 months. Abnormal brain MRI was observed in $53(83 \%)$ patients, with findings suggestive of mesial temporal sclerosis (MTS) accounting for $75 \%$ of these abnormalities. The presence of any MRI abnormality was associated with a 10 -month earlier tapering of AEDs $(p<0.01)$, and an 18-month earlier complete withdrawal of AEDs $(p<0.01)$. The odds of being seizure-free within the first year were higher if MTS was present in the brain MRI (adjusted $\mathrm{OR}=16$ ). Similarly, this was associated with seizure freedom after the first year (adjusted OR=14.8, $p<0.01$ ). The presence of unilateral temporal IEDs on preoperative EEG were also associated with earlier tapering and discontinuation of AEDs as well as a seizure-free state after ATL surgery $(\mathrm{OR}=8.5$ and 4.2 , for the first and second year respectively, $p<0.01$ ).

Conclusion: Patients with abnormal MRI findings and unilateral IEDs had earlier tapering of AEDs with an overall shorter AED discontinuation plan. Moreover, the presence of MTS on MRI, along with unilateral IEDs, were predictors of seizure freedom following ATL.

\section{Neurosciences 2021; Vol. 26 (3): 270-276 doi: 10.17712/nsj.2021.3.20200182}

From the Department of Neurosciences (Albalawi, Babtain, Baeesa, Al-Said, Alqadi), King Faisal Specialist Hospital and Research Centre, and from the Division of Neurosurgery (Baeesa), Department of Surgery, King Abdulaziz University, Jeddah, Saudi Arabia

Received 15th December 2020. Accepted 16th April 2021.

Address correspondence and reprint request to: Dr. Miad F. Albalawi, Department of Neurosciences, King Faisal Specialist Hospital \& Research Center, Jeddah, Saudi Arabia. E-mail: Miad.albalawi@gmail.com ORCID ID https://orcid.org/0000-0002-1489-1655 
A nterior temporal lobectomy (ATL) is the standard treatment for medically refractory mesial temporal lobe epilepsy (MTLE), achieving seizure remission in approximately $70 \%$ of patients. ${ }^{1-3}$ However, the feasibility and timing of antiepileptic drug (AEDs) withdrawal after ATL are debatable. ${ }^{4}$ The need for AEDs withdrawal stems from the adverse effects following long-term use, ${ }^{5,6}$ difficulties in maintaining compliance, and their high cost. ${ }^{7,8}$ Moreover, AEDs withdrawal following successful ATL is generally considered safe. ${ }^{4}$ There are no evidence-based guidelines for managing AEDs withdrawal after resective epilepsy surgery. ${ }^{9,10}$ Moreover, few prospective and retrospective studies have assessed postoperative AEDs management exclusively in patients with MTLE. ${ }^{411-13}$ Hence, the timing of AEDs withdrawal after temporal lobectomy is controversial. ${ }^{12,14,15}$ The difficulties faced are how to taper AEDs, how soon it is safe to taper, which clinical profiles favor tapering, and what is the optimal time to start the tapering process. Predicting successful AEDs withdrawal following ATL has been examined in a few studies; however, the results were inconsistent.9,12,16-18 In one study, brain magnetic resonance imaging (MRI) findings were associated with seizure outcomes following ATL with successful AEDs discontinuation. ${ }^{12}$ The MRI can detect brain abnormalities, predict postoperative seizure, and AEDs freedom ${ }^{2}$ Despite this, data are lacking regarding the role of MRI in determining the time to start tapering AEDs postoperatively, as well as the time until AEDs discontinuation in those who do not require postoperative AEDs treatment. These data are essential for establishing guidelines that could assist clinicians with AED management following ATL.

We aimed to assess the role of brain MRI in planning the tapering of AEDs and determining when to discontinue AEDs in patients with MTLE following ATL. We also evaluated seizure outcomes in patients with a minimum of 2 years of postoperative follow-up.

Methods. We conducted a retrospective chart review from January 2004 to December 2017 at the epilepsy center of King Faisal Specialist Hospital and Research Centre, Jeddah, Saudi Arabia.

In our epilepsy center and based on a structured institutional pathway, we discuss cases that are

Disclosure. The Authors have no conflict of interests, and the work was not supported or funded by any drug company. potential candidates for epilepsy surgery in our weekly epilepsy meetings with epileptologists and epilepsy neurosurgeons. In some cases, additional evaluation, such as fluorodeoxyglucose positron emission tomography and intracranial monitoring (e.g., subdural electrodes and intraoperative electrocorticography), is performed. We included adult patients with MTLE who underwent ATL between January 2004 and December 2017 who were followed up for a minimum 2 years postoperatively. Patients with incomplete medical records, more than one epilepsy surgery, or the resection extended beyond the standard anterior temporal lobe resection were excluded.

We collected the following data: basic demographics, age, age at the time of diagnosis, age at the time of surgery, presence of preoperative bilateral tonic-clonic seizure, preoperative seizure frequency, history of febrile convulsions (FC), preoperative 1.5-tesla or 3-tesla epilepsy protocol brain MRI and electroencephalography (EEG) results, use of invasive intracranial monitoring, type and side of surgery, pathology, number of medications preoperatively and postoperatively, and postoperative seizure recurrence if any. Regarding brain MRI, our epilepsy protocol involves the following sequences: sagittal T1, sagittal fast spoiled gradient echo, sagittal cube double inversion recovery white matter null, axial T1, axial T2-weighted fluid attenuated inversion recovery (FLAIR), axial T2, axial diffusion-weighted imaging, axial inversion recovery (IR), coronal T1, coronal T2-weighted FLAIR thin cuts, coronal T2 with 3-mm slice thickness, coronal IR, and coronal gradient echo sequences without contrast.

After resective epilepsy surgery, AEDs management was individualized for each patient by the treating epileptologists. We also collected data on the time (in months) required to start tapering of $\mathrm{AEDs}$ in those who were suitable candidates. We also determined the time until the patient was completely off AEDs, if applicable. We also identified seizure outcomes following epilepsy surgery at the end of the follow-up period. We divided patients into three groups according to their seizure control-seizure-free with an Engel class I outcome and off medications, seizure-free with an Engel class I outcome and on medications, and seizures on medications. The institutional review board approved this study (IRB 2020-24).

We used R Studio, R Core Team (2019) for statistical analysis (R: A language and environment for statistical computing, https://www.r-project.org). Data are presented as frequencies and percentages. Inferential statistics, including t-tests, linear regression, 
Table 1 - Seizure outcomes after anterior temporal lobectomy.

\begin{tabular}{|c|c|c|c|c|}
\hline Characteristic & $\begin{array}{c}\text { No seizures off } \\
\text { medication } \\
n=23\end{array}$ & $\begin{array}{c}\text { No seizures } \\
\text { on medication } \\
n=24\end{array}$ & $\begin{array}{c}\text { Seizures } \\
\text { on medication } \\
\mathrm{n}=17\end{array}$ & $P$-value \\
\hline Sex (male) & $16(70 \%)$ & $11(46 \%)$ & $12(71 \%)$ & NS \\
\hline Mean age at epilepsy surgery & 33 years & 36 years & 32 years & NS \\
\hline Baseline median of monthly seizures & 4 & 4 & 4.25 & NS \\
\hline Presence of febrile convulsions & $11(48 \%)$ & $6(25 \%)$ & $5(29 \%)$ & NS \\
\hline Median number of AEDs & 2 & 2.5 & 2 & NS \\
\hline Mean time to start tapering AEDs & 7 months & 10 months & 24 months & $<0.01$ \\
\hline Mean time to discontinue AEDs & 24 months & 60 months & 72 months & $<0.01$ \\
\hline MRI (Abnormal) & $23(100 \%)$ & $21(88 \%)$ & $9(53 \%)$ & $<0.01^{1}$ \\
\hline EEG (Unilateral IEDs) & $21(91 \%)$ & $16(67 \%)$ & $9(53 \%)$ & $<0.01^{2}$ \\
\hline MTS in pathology & $15(65 \%)$ & $14(58 \%)$ & $11(65 \%)$ & NS \\
\hline
\end{tabular}

chi-square, and analysis of variance, were used for statistical analyses of MRI findings, EEG results, other factors influencing the time to start tapering AEDs, the time required to complete tapering, and seizure recurrence. We determined 95\% confidence intervals and interpreted $p$-values according to the American Statistical Association guidelines. ${ }^{19}$

Results. Baseline characteristics. We studied 64 patients. There were 39 (61\%) men, the mean age at epilepsy onset was 12 years (standard deviation $[S D]=9.3$ years, range: $1-36$ years), and the mean age at epilepsy surgery was 34 years $(\mathrm{SD}=8.9$ years, range: 16-54 years). The FC was reported in 22 (34\%) patients. The median baseline number of monthly seizures was 4 ( $\mathrm{SD}=12.6$ seizures, interquartile range: $2-7$ seizures). We documented focal to bilateral tonic-clonic seizures in 44 patients (69\%) before surgery. Patients used a median of 2 AEDs at the time of epilepsy surgery. Brain MRI findings were abnormal in 53 (83\%) patients, with mesial temporal sclerosis (MTS) accounting for $75 \%$ of all MRI abnormalities (Figure 1). Scalp EEG before epilepsy surgery revealed abnormal findings in all but one patient; unilateral temporal interictal epileptic discharges (IEDs) were observed in 46 (72\%) cases, and bilateral temporal IEDs were reported in 17 $(27 \%)$ cases. All patients underwent anterior mesial temporal lobectomy as per the standard epilepsy surgery protocol. Histopathology showed findings consistent with hippocampal sclerosis in 40 (63\%) patients, ischemic changes and gliosis in eight (13\%) patients, nonspecific gliosis in nine (14\%) patients, focal cortical dysplasia (FCD) type IIIa in five $(8 \%)$ patients, FCD $1 \mathrm{a}$ in $2(3 \%)$ patients, and poorly classified pathological changes of uncertain significance in three $(5 \%)$ patients.
Table 1 shows the patient characteristics according to seizure outcomes.

Timing of tapering and discontinuing AEDs. All patients, except one, had their medications tapered. The mean time required to start tapering AEDs after ATL surgery was 10 months ( $\mathrm{SD}=13$ months, range: 1-72 months). We discontinued AEDs in 23 (35\%) patients, which took a mean duration of 48 months ( $S D=22$ months, range: 6-72 months). Patients with a brain MRI abnormality had a significantly shorter time to start AEDs tapering than those with no brain MRI abnormality (12 months vs. 22 months, $p<0.01$; 95\% confidence interval [CI], 2-18 months). This effect was not observed in multivariate analysis. Moreover, all seizure-free patients who were off medications had abnormal brain MRI findings and the earliest tapering of AEDs. In contrast, MRI abnormalities were detected in only $50 \%$ patients who continued to experience seizures and were on medications. They also had the longest duration to start tapering AEDs (Table 1).

In addition, it took an average of 18 months to discontinue AEDs in patients with an abnormality detected on brain MRI compared to those with no abnormality on brain MRI ( 44 vs. 62 months, $p<0.01$, 95\% CI: 4-25 months). This effect was also observed in the multiple linear regression analysis (18 months earlier, $p=0.05$, 95\% CI: $1-37$ months) after adjusting for gender, age at surgery, EEG and pathology findings, baseline number of seizures, and the presence of FC. The overall observations suggested a significant role of brain MRI in determining the time to taper off and discontinue AEDs following ATL. Unilateral temporal IEDs were associated with a 7-month earlier tapering of AEDs than other EEG findings (12 vs. 19 months, $p<0.05$, 95\% CI: $1-14$ months), but this effect was not 


\section{Brain MRI findings}

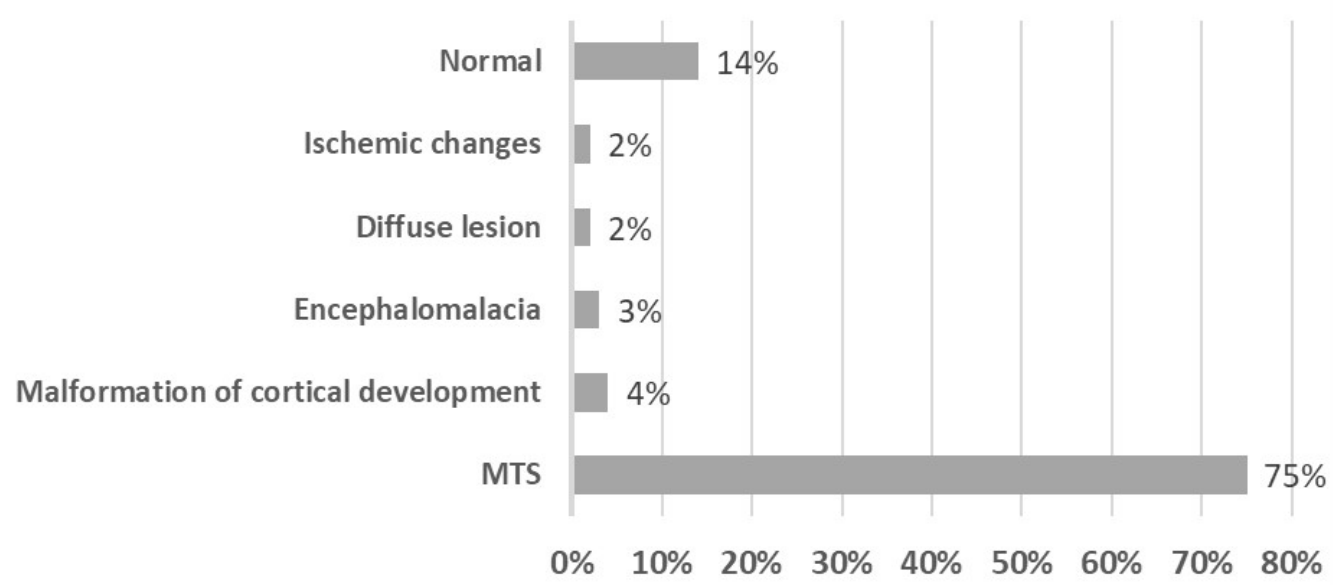

Figure 1 - Characteristics of MRI brain findings.

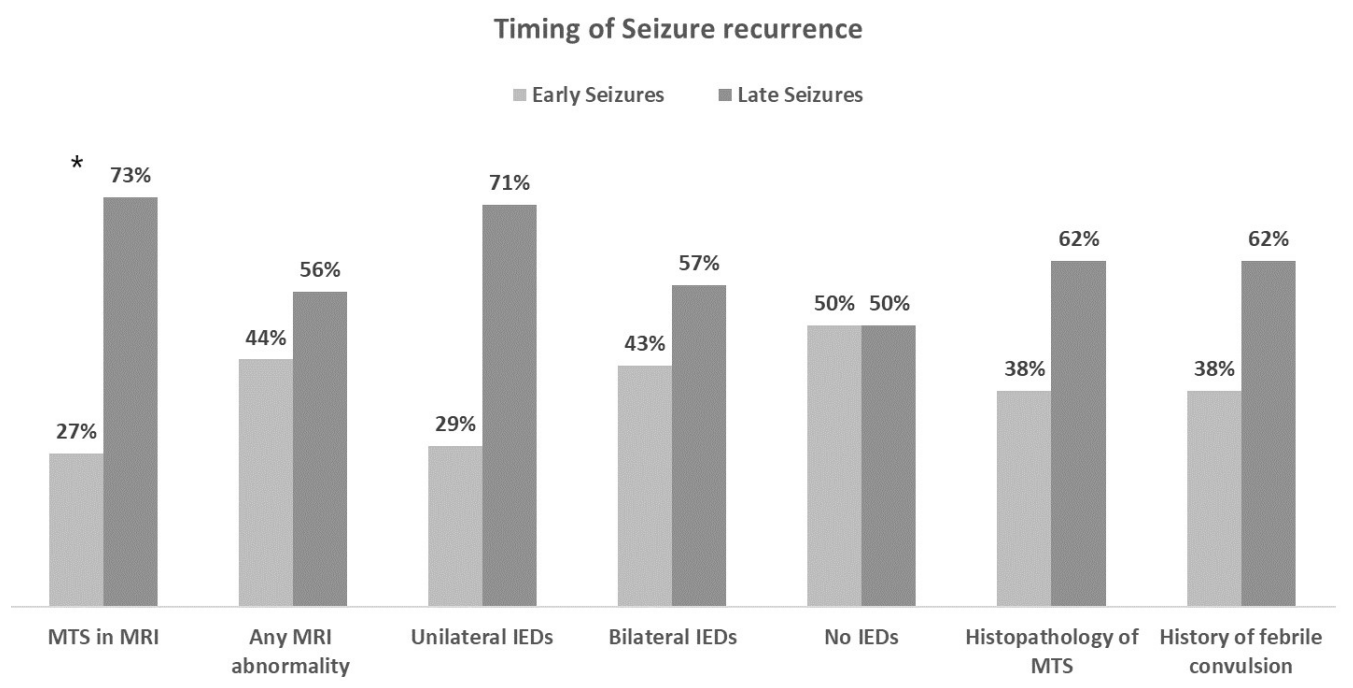

Figure 2 - Correlation of seizures retiming of seizures and patient characteristics $\left({ }^{*}\right.$ indicated $p$-value $\left.<0.05\right)$.

present in the multiple linear regression analysis. On further analysis, $91 \%$ of seizure-free patients who were off AEDs had unilateral IEDs. They also had a shorter duration to the start of AEDs tapering than patients with other EEG discharge patterns (6 vs. 9 months, $p<0.05$, 95\% CI: $1-7$ months). These observations suggest an association between these EEG patterns and a favorable prognosis.

Finally, the time to discontinue AEDs was influenced significantly by EEG discharge pattens; it was shorter in patients who had unilateral temporal IEDs than in those who had bilateral IEDs (47 vs. 64 months, $p<0.01$, 95\% CI: 5-29 months). There was no combined effect of MRI and EEG patterns on the time to tapering or discontinuing AEDs.

Seizure recurrence and outcomes. A total of 28 (44\%) patients had seizures after ATL, of whom 16 (25\%) patients had recurrent seizures within the first year. Figure 2 shows the timing of seizure recurrence and patient characteristics. When comparing early versus late seizures, only the presence of MTS on brain MRI showed a significant association with seizure recurrence timing, and was associated with a reduced likelihood of seizures within the first year postoperatively $(\mathrm{OR}=0.22$, $p<0.05$, 95\% CI: 0.06-0.89). Furthermore, multiple 
logistic regression analysis was performed to predict seizures within the first year. The absence of seizures within the first year was significantly associated with the presence of MTS on brain MRI (adjusted OR= 16, $p<0.01,95 \%$ CI: 2.3-166.4). Unilateral IEDs on preoperative EEGs were also significant predictors of seizure freedom within the first year (adjusted $\mathrm{OR}=8.5$, $p<0.01,95 \% \mathrm{CI}: 1.8-51.2)$. Brain MRI findings and EEG patterns were also significant predictors of seizure freedom (adjusted OR for MTS on brain MRI=14.8, $p<0.01,95 \%$ CI: 2.3-29.7; adjusted OR for unilateral IEDs $=4.2, \quad p<0.01,95 \%$ CI: 1.2-17.2). These observations were adjusted for age at surgery, gender, febrile seizures, MRI pathology, and baseline number of seizures before surgery.

By the end of the follow-up, seizure freedom, irrespective of being on or off medications, was achieved in $45(70.3 \%)$ patients. This state was best predicted by the presence of an MRI lesion suggestive of MTS $(\mathrm{OR}=32.3, p<0.01,95 \% \mathrm{CI}: 4.5-69.1)$ after adjusting for EEG and pathology findings, baseline number of seizures, gender, and presence of FC. Bivariate analysis also showed an association between seizure freedom and brain MRI abnormalities (crude OR=19.3, $p<0.01$, 95\% CI: 3.61-103.78).

Discussion. Our study comprised a group of patients with refractory MTLE; most of our study population had brain MRI features suggestive of MTS, and all patients underwent a standard protocol for ATL at our center. Up to $70 \%$ of our patients achieved seizure freedom, and approximately one-third (35.9\%) of the patients were off AEDs at the final follow-up. Our findings are similar to those of previously published reports. ${ }^{9,11,16,18,20-22}$ Hence, the feasibility and safety of AEDs withdrawal after successful ATL have been confirmed in this population. ${ }^{4,17}$ It is a common practice to wait for one or 2 years of seizure freedom before attempting any medication change after epilepsy surgery. ${ }^{9,11,23-25}$ These practices are influenced by the fact that most seizure recurrences occur in the first 12-24 months postoperatively; ${ }^{17,26}$ however, it leaves patients exposed to the continued adverse effects and costs of AEDs. ${ }^{7,8}$

In our cohort, patients received individualized postoperative management of AEDs based on the treating epileptologist's decisions, considering the number of AEDs, their side effect profile, pathology results, postoperative EEG findings, brain MRI results, and the patient's desires. However, our study showed that brain MRI findings significantly influenced the timing of AEDs withdrawal; their presence allowed for a 10-month earlier tapering of AEDs. In addition, patients who were seizure free and off medications by the end of the study had the shortest time between surgery and initiation of AEDs tapering, and all these patients had abnormal brain MRI findings. Our study also demonstrated a significant effect of EEG findings on the timing of AEDs withdrawal and postoperative discontinuation. This association between brain MRI findings and EEG discharges and the postoperative management of AEDs has not been reported in previous studies. Following strict protocols for the management of AEDs post-epilepsy surgery in some centers could mask such an association. ${ }^{4,12,16}$

In contrast, the time to discontinuation of AEDs was significantly shorter in patients who had brain MRI abnormalities and in those with unilateral IEDs. This duration was shorter by $50 \%$ in patients who ultimately achieved a seizure-free state and continued to be off medications, all of whom had abnormal MRI findings before epilepsy surgery. These results, shows a new role for the use of MRI and EEG in AEDs management post-ATL, which could guide epileptologists in managing AEDs postoperatively.

Abnormal brain MRI findings predicted favorable postoperative seizure outcome as well as successful AEDs discontinuation. ${ }^{2,12,24}$ In our group, 70\% patients achieved a seizure-free state postoperatively; brain MRI abnormalities were detected in $80-100 \%$ of these patients. Brain MRI abnormalities were a predictor of favorable postoperative seizure control, irrespective of whether the patient continued to use AEDs. Specifically, the presence of MTS on brain MRI was associated with an approximately 30 times increase in the likelihood of seizure freedom by the end of follow-up in our patients. In one study, the authors advised a cautious withdrawal of AEDs following epilepsy surgery in patients with MTLE, normal MRI finding, and without HS on pathology as they were more prone to seizure recurrence. ${ }^{4}$ We found that the appearance of MTS on brain MRI was a significant predictor of seizure freedom within the first year postoperatively and afterward. Despite the presence of HS in 29 (45\%) patients in our group who were seizure-free at the end of the study, we did not find an association between histopathological findings and AEDs tapering. We also did not find any association between HS and seizure outcomes, similar to previously published studies. ${ }^{12,18}$

Unilateral IEDs before surgery were a predictive factor for seizure freedom within the first year and were associated with an absence of late seizure recurrence 
after resection. This is consistent with the findings of previous studies. ${ }^{21,24} \mathrm{We}$ also found that patients who continued to have seizures had more bilateral IEDs than seizure-free patients, consistent with the findings of previous reports. ${ }^{27,28}$

Our study has a few limitations. Although it provides insights into the crucial issue of AEDs management after ATL, the sample size was relatively small, and the study was conducted retrospectively. Prospective studies with a larger sample size are needed. Follow up EEG after resective epilepsy surgery is not performed routinely in all patients at our center; thus, it was not performed in every patient in this cohort, which limited the availability of postoperative EEG data that could be assessed in relation to AEDs management following epilepsy surgery in this study. We acknowledge the prognostic value of the latter for AEDs withdrawal in patients with MTLE. ${ }^{12}$ We also realize that individualized AEDs management after resection, rather than following a standard protocol, may hinder the generalizability of AEDs withdrawal. Nevertheless, our practice led to this study's findings and demonstrated the role of brain MRI and EEG findings in AEDs management after ATL in patients with intractable epilepsy.

In conclusion, AEDs withdrawal after ATL is complex, yet successful discontinuation is possible. Careful selection of patients who would benefit from earlier AEDs withdrawal can be considered based on clinical factors, particularly in those with abnormal MRI findings and unilateral temporal IEDs. This study demonstrates the effects of MRI and EEG findings on the timing of AEDs withdrawal. Future prospective studies are needed to validate these findings and help determine the safe timing for AEDs tapering after ATL epilepsy surgery.

Acknowledgements. The authors would like to thank editage for English language editing.

\section{References}

1. Wiebe S, Blume WT, Girvin JP, Eliasziw M. A randomized, controlled trial of surgery for temporal-lobe epilepsy. $N$ Engl J Med 2001; 345: 311-318.

2. McIntosh A, Wilson SJ, Berkovic SF. Seizure outcome after temporal lobectomy: current research practice and findings. Epilepsia 2001; 42: 1288-1307.

3. Téllez-Zenteno JF, Dhar R, Wiebe S. Long-term seizure outcomes following epilepsy surgery: a systematic review and meta-analysis. Brain 2005; 128: 1188-1198.

4. Rathore C, Panda S, Sarma PS, Radhakrishnan K. How safe is it to withdraw antiepileptic drugs following successful surgery for mesial temporal lobe epilepsy? Epilepsia 2011; 52: 627-635.
5. Cramer JA, Mintzer S, Wheless J, Mattson RH. Adverse effects of antiepileptic drugs: a brief overview of important issues. Expert Rev Neurother 2010; 10: 885-891.

6. Hoppe C, Poepel A, Sassen R, Elger CE. Discontinuation of anticonvulsant medication after epilepsy surgery in children. Epilepsia 2006; 47: 580-583.

7. Ivanova JI, Birnbaum HG, Kidolezi Y, Qiu Y, Mallett D, Caleo S. Economic burden of epilepsy among the privately insured in the US. Pharmacoeconomics 2010; 28: 675-685.

8. Langfitt J, Holloway R, McDermott M, Messing S, Sarosky K, Berg A, et al. Health care costs decline after successful epilepsy surgery. Neurology 2007; 68: 1290-1298.

9. Al-Kaylani M, Konrad P, Lazenby B, Blumenkopf B, Abou-Khalil B. Seizure freedom off antiepileptic drugs after temporal lobe epilepsy surgery. Seizure 2007; 16: 95-98.

10. Schmidt D, Baumgartner C, Löscher W. Seizure recurrence after planned discontinuation of antiepileptic drugs in seizurefree patients after epilepsy surgery: a review of current clinical experience. Epilepsia 2004; 45: 179-186.

11. Lee S-Y, Lee J-Y, Kim DW, Lee SK, Chung CK. Factors related to successful antiepileptic drug withdrawal after anterior temporal lobectomy for medial temporal lobe epilepsy. Seizure 2008; 17: 11-18.

12. Rathore C, Jeyaraj MK, Dash GK, Wattamwar P, Baheti N, Sarma SP, et al. Outcome after seizure recurrence on antiepileptic drug withdrawal following temporal lobectomy. Neurology 2018; 91: e208-e216.

13. Pimentel J, Peralta AR, Campos A, Bentes C, Ferreira AG. Antiepileptic drugs management and long-term seizure outcome in post surgical mesial temporal lobe epilepsy with hippocampal sclerosis. Epilepsy Res 2012; 100: 55-58.

14. Ladino LD, Hernández-Ronquillo L, Téllez-Zenteno JF. Management of antiepileptic drugs following epilepsy surgery: a meta-analysis. Epilepsy Res 2014; 108: 765-774.

15. Cole AJ, Wiebe S. Debate: should antiepileptic drugs be stopped after successful epilepsy surgery? Epilepsia 2008; 49: 29-34.

16. Maehara T, Ohno K. Preoperative factors associated with antiepileptic drug withdrawal following surgery for intractable temporal lobe epilepsy. Neurol Med Chir (Tokyo) 2011; 51: 344-348.

17. Yardi R, Irwin A, Kayyali H, Gupta A, Nair D, Gonzalez Martinez J, et al. Reducing versus stopping antiepileptic medications after temporal lobe surgery. Ann Clin Transl Neurol 2014; 1: 115-123.

18. Kim YD, Heo K, Park SC, Huh K, Chang JW, Choi JU, et al. Antiepileptic drug withdrawal after successful surgery for intractable temporal lobe epilepsy. Epilepsia 2005; 46: 251-7.

19. Wasserstein RL, Lazar NA. The ASA Statement on p-Values: Context, Process, and Purpose. The American Statistician 2016; 70: 2: 129-133.

20. Lowe AJ, David E, Kilpatrick CJ, Matkovic Z, Cook MJ, Kaye A, et al. Epilepsy surgery for pathologically proven hippocampal sclerosis provides long-term seizure control and improved quality of life. Epilepsia 2004; 45: 237-242.

21. McIntosh AM, Kalnins RM, Mitchell LA, Fabinyi GC, Briellmann RS, Berkovic SF. Temporal lobectomy: long-term seizure outcome, late recurrence and risks for seizure recurrence. Brain 2004; 127: 2018-2030.

22. Wieser HG, Häne A. Antiepileptic drug treatment before and after selective amygdalohippocampectomy. Epilepsy Res 2003; 55: 211-223. 
23. Berg AT, Langfitt JT, Spencer SS, Vickrey BG. Stopping antiepileptic drugs after epilepsy surgery: a survey of US epilepsy center neurologists. Epilepsy Behav 2007; 10: 219-222.

24. Radhakrishnan K, So EL, Silbert P, Jack C, Cascino GD, Sharbrough F, et al. Predictors of outcome of anterior temporal lobectomy for intractable epilepsy: a multivariate study. Neurology 1998; 51: 465-471.

25. Jeong SW, Lee SK, Hong KS, Kim KK, Chung CK, Kim H. Prognostic factors for the surgery for mesial temporal lobe epilepsy: longitudinal analysis. Epilepsia 2005; 46: 1273-1279.
26. Ramesha KN, Mooney T, Sarma PS, Radhakrishnan K. Long-term seizure outcome and its predictors in patients with recurrent seizures during the first year aftertemporal lobe resective epilepsy surgery. Epilepsia 2011; 52: 917-924.

27. Kelemen A, Barsi P, Erőss L, Vajda J, Czirják S, Borbély C, et al. Long-term outcome after temporal lobe surgery-prediction of late worsening of seizure control. Seizure 2006; 15: 49-55.

28. Elsharkawy AE, Alabbasi AH, Pannek H, Oppel F, Schulz $\mathrm{R}$, Hoppe $\mathrm{M}$, et al. Long-term outcome after temporal lobe epilepsy surgery in 434 consecutive adult patients. J Neurosurg 2009; 110: 1135-1146.

\section{Authorship entitlement}

Excerpts from the Uniform Requirements for Manuscripts Submitted to Biomedical Journals updated November 2003.

Available from www.icmje.org

The international Committee of Medical Journal Editors has recommended the following criteria for authorship; these criteria are still appropriate for those journals that distinguish authors from other contributors.

Authorship credit should be based on 1) substantial contributions to conception and design, or acquisition of data, or analysis and interpretation of data; 2) intellectual content; and 3) final approval of the version to be published. Authors should meet conditions 1,2 , and 3 .

Acquisition of funding, collection of data, or general supervision of the research group, alone, does not justify authorship.

An author should be prepared to explain the order in which authors are listed. 